InnOvaciOnes de NegOciOs 17(34): 169-189

(C) 2020 UANL, Impreso en México (ISSN: 2007-1191)

Recepción: 6 de mayo 2020 Aceptación: 16 de junio 2020

\title{
Variables relacionadas al rendimiento en el examen general para el Egreso de Licenciatura de Arquitectura, caso Universidad Autónoma de Nuevo León (Variables related to performance in the general exam for the Bachelor's Degree in Architecture, Autonomous University of Nuevo Leon case)

\author{
Miguel Angel Fitch Osuna, María de Jesús Araiza Vázquez \\ Universidad Autónoma de Nuevo Leon, Facultad de Contaduría Pública y \\ Administración \\ miguelfosuna@gmail.com,maria.araizavz@uanl.edu.mx
}

\begin{abstract}
The purpose of this research revolved around the factors related to the student with respect to the evaluation of the exit exam in the Bachelor of Architecture, the results of the evaluations were interpreted during the course of his degree, taking into account from his access score to the degree, the final average and other variables that could be correlated with the four areas of knowledge of the General Exam for the Exit of the Degree (EGEL). This in order to make suggestions to the academy that allow it to focus efforts and carry out academic-administrative strategies that help achieve improvements in the results of the students and that this same I achieved the necessary skills for their graduation. Within the results, it was highlighted that the access score is correlated with the EGEL average, the general average, as well as the different areas of knowledge except for the Architectural Project (PA) area, for this reason, the different recommendations are They directed towards this practical exam, which turned out to obtain the lowest results in the knowledge areas of the aforementioned evaluation.
\end{abstract}

Keywords: EGEL, Arquitectura, Puntaje de acceso, Promedio

JEL: I2, I21, I23

Variables relacionadas al rendimiento en el examen general de egreso 
Resumen: El propósito de esta investigación giró alrededor de los factores relacionados al estudiante con respecto a la evaluación del examen de egreso en la licenciatura de Arquitectura, se interpretaron los resultados de las evaluaciones durante la trayectoria de su licenciatura, tomando en cuenta desde su puntaje de acceso a la licenciatura, el promedio final y otras variables que se pudiesen correlacionar con las cuatro áreas de conocimiento del Examen General para el Egreso de la Licenciatura (EGEL). Esto con el fin de realizar sugerencias a la academia que le permita enfocar esfuerzos y realizar estrategias académicas-administrativas que ayuden a lograr mejoras en los resultados de los estudiantes y que este mismo logré las competencias necesarias para su egreso. Dentro los resultados se destacaron que el puntaje de acceso se encuentra correlacionado sobre el promedio del EGEL, el promedio general, así como de las distintitas áreas de conocimiento a excepción del área de Proyecto Arquitectónico (PA), por este motivo, las distintas recomendaciones se dirigieron hacia este examen práctico, el cual resultó obtener los resultados más bajos en las áreas de conocimiento de la antes mencionada evaluación.

Palabras clave: EGEL, Arquitectura, Puntaje de acceso, Promedio.

\title{
Introducción
}

Dentro de los márgenes de competitividad que exige la globalización, las instituciones de nivel superior mantienen dentro de sus principales objetivos cumplir con los distintos requerimientos que le aplican las acreditadoras internacionales, así como los organismos de evaluación, ya que esto implica de cierta manera las condiciones que los estudiantes enfrentan al medio laboral en el momento de egresar, para tal finalidad se establecen indicadores tales como el rendimiento académico, resultados de examen de egreso, entre otros.

En este sentido una forma en que las universidades miden el nivel de competencias logradas 0 identifican el nivel de conocimientos y habilidades académicas de los egresados es mediante el Examen General para el Egreso de Licenciatura (EGEL), los resultados de esta prueba no han sido de todo

\author{
Fitch, M. \& Araiza, M.
}


satisfactorios desde sus inicios a la fecha, dejando una brecha de oportunidad en el quehacer educativo. En el caso de la carrera de arquitectura y los resultados 2017 , muestran que solo un $4.6 \%$ de los sustentantes lograron testimonio sobresaliente, $9.2 \%$ desempeño satisfactorio y un $86.2 \%$ no obtuvo testimonio (Centro Nacional de Evaluación para la Educación Superior, 2018, pág. 28).

Estos resultados producen la inquietud de buscar las causas que originan tan bajos niveles de cumplimiento siendo fundamental que se presenten alternativas de estudio para detectar aquellos factores que se encuentren relacionados con estos resultados, de esta manera, incidir en la toma de decisiones del personal involucrado que intervienen en los procesos académicos, para que se utilicen de manera efectiva los esfuerzos y recursos relacionados con el aprendizaje.

La Facultad de Arquiectura de la Universaidad Autonoma de Nuevo León, no está excenta de esta inquietud, a la fecha cuenta con una población aproximada de 4181 estudiantes en la licenciatura de Arquitectura de los cuales presentan el examen de egreso entre 220 a 330 estudiantes por semestre, siendo una cantidad considerable aquellos que no logran los resultados satisfactorios, es por ello que uno de los principales objetivos de esta investigación es encontrar algún indicio que nos permita determinar los factores de éxito en el examen de egreso.

Sin embargo, el éxito académico basado en el rendimiento universitario, tiene una amplia variedad de componentes que van desde las habilidades cognitivas, intereses, motivación, autoconcepto, contexto socioeconómico, contexto socio histórico, dinámica familiar, salud, ambiente escolar, influencia de los padres y compañeros, escolaridad de los padres, características de quien enseña y como lo hace, hasta variables relacionadas con los programas y el currículo (Cortés Flores \& Palomar Lever, 2008, pág. 198).

En la investigación a estudiantes de la licenciatura de Psicología realizada por Elías, Caldera, Reynoso y Zamora (2016), se tomó como objetivo identificar las relaciones entre tipo de ingreso, sexo, puntuación de examen de aptitud académica, los examenes de ingreso y egreso de los estudiantes.

\section{Variables relacionadas al rendimiento en el examen general de egreso}


Resultando correlaciones importantes entre los resultados de egreso y el puntaje de aptitud, así como correlación entre el promedio de bachillerato con el promedio general de egreso, explicando que esta relación se obtiene ya que las calificaciones que emiten los profesores en ambos niveles consideran aspectos que no pueden ser valorados en pruebas objetivas 0 examenes, tal es el caso de la participación, orden, disciplina, puntualidad, disposición al trabajo entre otros; mientras que los examenes de puntaje influyen más las estructuras cognitivas y los conocimientos previos (págs. 85-86).

De manera similar, Izar y Espericueta (2013), realizarón un análisis estadístico de regresión logistica a los datos referentes a estudiantes de la carrera de Mecánico Administrador de la Universidad Autónoma de San Luis Potosí, a partir de la correlación con los resultados del Examen de Egreso, determinando que el lugar ocupado en el examen de admisión y el promedio general son las variables predictivas hacia el desempeño en dicha evaluación.

Por su parte, Cortés y Palomar (2008), tuvieron como objetivo de su investigación conocer la validez predictiva del proceso de admisión en el rendimiento académico con respecto al primer año de licenciatura, para ello se tomó la información de 240 estudiantes de la carrera de Psicología de una universidad privada de la Ciudad de México y como variables predictoras se consideró las calificaciones en el Examen Nacional de Ingreso a la Educación Superior (EXANI-II), el promedio general de preparatoria y el puntaje obtenido en el cuestionario sobre problemas sociales (DIT), encontrandosé un predominio de correlaciones bajas y moderadas entre el promedio del EXANIII, el promedio del bachiderato, el desarrollo moral, el puntaje de la Universidad Iberoamericana de México y el rendimiento académico en el primer año de la carrera de Psicología, concluyendo que son muchas variables involucradas con el rendimiento académico a lo que se requieren más investigaciones multicausales que expliquen a profundidad este fenómeno.

En el caso de la aplicación del EGEL en medicina Guerrero, Romero y Noriega (2013), encuentran que los estudiantes que tuvieron una edad menor a 30 años al momento de egresar y un promedio de salida de la universidad de 9 o mayor, estos poseen una mejor probabilidad de obtener un resultado satisfactorio o sobresaliente (pág. 35).

\section{Fitch, M. \& Araiza, M.}


En cuanto a los resultados obtenidos por Izar y López (2009), aplicados a 47 estudiantes de la Universidad de San Luis Potosí en la carrera de Contaduría Pública, se mostró que los alumnos que obtienen mayores promedios durante la licenciatura y en las materias de fiscal y contabilidad, logran mejores resultados en los exámenes de egreso, aclaran además, que el análisis de regresión no dio un ajuste satisfactorio así que no se puede usar con fines predictivos (págs. 32-33).

Los resultados de la investigación realizada por Toscano, Ponce, Margain y Vizacaíno (2016), nos indican que para un total de 22,367 sustentantes de licenciatura en informática, del año 2008 al 2014, las variables institución de procedencia y el promedio son las que guardan una mayor correlación sin llegar a ser de manera perfecta a lo que se explorará otras técnicas para el análisis de datos con variables que esten más relacionadas con su formación y trayectoria académica (pág. 81).

Analizando las invetigaciones exstentes, se considera la siguiente pregunta central de investigación:

¿El resultado del examen de ingreso a la licenciatura, el promedio durante la trayectoria académica, el grado de estudio de los padres, el tiempo de experiencia, así como el promedio de algunas unidades de aprendizaje en particular se correlacionan positivamente en los resultados del examen de perfil de egreso?

Como hipótesis especificas tenemos:

- El resultado del examen de ingreso a la UANL tiene una correlación significativamente positiva con los resultados del perfil de egreso.

- El promedio general en la licenciatura (Promaprobadas) tiene una correlación significativamente positiva con los resultados del perfil de egreso.

- El Promedio materias relacionadas con EGEL (Promaterias) tiene una correlación significativamente positiva con los resultados del perfil de egreso.

\section{Variables relacionadas al rendimiento en el examen general de egreso}


- El Promedio (Promedio), guarda una correlación significativamente positiva con los resultados del perfil de egreso.

- El grado de estudio de los padres tiene una correlación significativamente positiva con los resultados del perfil de egreso.

- El Tiempo experiencia profesional relacionado con la licenciatura (Expro) tiene una correlación significativamente positiva con los resultados del perfil de egreso.

En base a lo revisado en la lieratura y las aportaciones empiricas de los antes mencionados investigadores, se representó gráficamente las hipótesis especificas (Ilustración 1), podiendo observar las correlaciones que se plantean entre las distintas variables independientes y el examen de EGEL.

llustración 1. Representación gráfica de la hipótesis

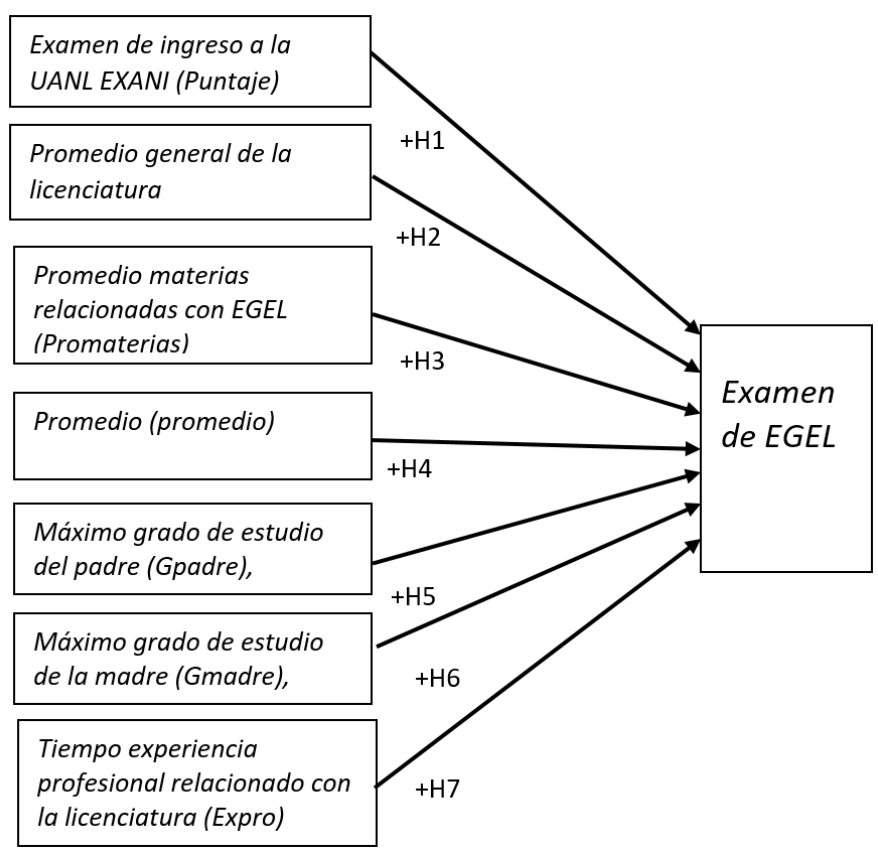

Fuente: Elaboración propia

Fitch, M. \& Araiza, M. 


\section{Metodología}

Esta investigación corresponde al tipo cuantitativa, descriptiva con diseño no experimental y transversal, además de buscar las correlaciones entre variables. En un inició se realizó revisión de la literatura especializada con el tema de interés, consultando artículos relacionados con exámenes de egreso y las variables circunscritas al rendimiento académico a nivel superior.

En base a esta búsqueda se tomaron en cuenta las siguientes variables:

Examen de EGEL (PromEGEL), examen de cobertura nacional que evalúa los conocimientos y habilidades necesarios de los recién egresados para su inserción al ejercicio profesional, consta del promedio de cuatro áreas, como a continuación se explica.

Proyecto de espacios habitables con enfoque sustentable (PEHES), consta de la elaboración del marco teórico-conceptual del problema de hábitat de usuario, identificación de las características y necesidades del usuario, análisis del contexto del problema a resolver y la formulación del programa arquitectónico.

Construcción de espacios habitables sustentables (CEHS), se basa en el desarrollo del sistema constructivo, desarrollo de instalaciones, desarrollo de la estructura y el desarrollo del proyecto ejecutivo.

Administración y gestión de la construcción (AGC), aborda preguntas acerca de la gestión para materializar el proyecto y la adminisración de los servicios de arquitectura.

Proyecto arquitectónico (PA), a diferencia de las tres primeras que se aplica un examen de opción múltiple, está cuarta corresponde a la elaboración de un anteproyecto arquitectónico. Esta última, con la finalidad de evaluar otro tipo de habilidades complejas propias de la profesión que no son susceptibles de ser evaluadas mediante los otros instrumentos. Existen tres niveles de desempeño en base a la puntuación obtenida: Aún no satisfactorio (700-999), desempeño satisfactorio (1000-1149) y desempeño sobresaliente (11501300) (Centro Nacional de Evaluación para la Educación Superior, 2018, págs. 12-13).

\section{Variables relacionadas al rendimiento en el examen general de egreso}


Examen de ingreso a la UANL EXANI (Puntaje), se tomó la evaluación obtenida en el examen de selección para el ingreso a la licenciatura, el cual evalúa el nivel de logro de los aspirantes para reconocer, comprender, resolver e interpretar planteamientos en los que debe de aplicar los conocimientos y las habilidades adquiridos durante la educación media superior (Centro Nacional para la Educación Superior, A.C., 2019).

Promedio general en la licenciatura (Promaprobadas), se tomó el promedio de resultado aprobatorio de todas las unidades de aprendizaje cursadas durante la licenciatura.

Promedio materias relacionadas con EGEL (Promaterias), se tomo en cuenta el promedio de las unidades de aprendizaje que se encuentran más relacionadas a preparar al estudiante para el EGEL, tal como: Taller integral 1, Taller integral 2, Representación gráfica asistida por computadora, Taller de proyecto ejecutivo 2 y Taller de proyecto ejecutivo 3

Promedio (promedio), esta variable se tomó de la base de datos SIASE, la cual toma en cuenta los resultados de todas las oportunidades de cada una de las unidades de aprendizaje cursadas por el estudiante.

Género: masculino o femenino, a final de analizar si existen diferencias siginificativas por genero, se tomó en cuenta esta variable.

Máximo grado de estudio del padre (Gpadre), nivel de grado de estudio alcanzado por el padre, tomando en cuenta desde secundaria hasta posgrado.

Máximo grado de estudio de la madre (Gmadre), último grado de estudios alcanzado por la madre.

Tiempo experiencia profesional relacionado con la licenciatura (Expro), el tiempo que el estudiante ha desempeñado algún puesto de trabajo relacionado exclusivamente con su licenciatura.

Primeramente, se solicitó a las autoridades de la Facultad de Arquitectura de la UANL, los permisos y autorizaciones tanto como para aplicar algún tipo de instrumento, así como para consulta de bases de datos institucionales. Posteriormente se adquirió información de los estudiantes que se encontraban en su último semestre de licenciatura y próximos a presentar examen de egreso (EGEL), se validó la población y se calculo la muestra, la cual resultó en 109 estudiantes de Arquitectura los cuales se seleccionaron de

\author{
Fitch, M. \& Araiza, M.
}


manera aleatoria. Aprovechando una investigación paralela con objetivos distintos, se tomaron en cuenta la información recolectada del instrumento de aprovechamiento de recursos tecnológicos, el cual toma en cuenta algunas variables de interés a esta investigación. Una vez aplicado el instrumento se consultó la base datos de la UANL (SIASE), para obtener las variables restantes. Para el análisis estadístico descriptivo, diferencias de medias y correlación de variables, se utilizó la aplicación de IBM SPSS.

\section{Resultados}

Una vez definidas las variables en base a la revisión de la literatura se procedió a analizar los datos con el programa estadístico IBM SPSS. Primero se analizó su posible uso en pruebas parametricas, para el caso de la normalidad se utilizó el test de Kolmogorov-Smirnov y para la homogeniedad o homocedasticidad se realizó el test de Levene.

Realizando la prueba de Kolmogorov-Smirnof, podemos apreciar en la Tabla 1, que todas las variables tienen una distribución normal por contar con un nivel de significancia ( $p>.05$ ), además observamos en la llustración 2, que las variables PromEGEL, Puntaje y Promedio; se comportan dentro de los parametros de una distribución normal.

Tabla 1 Prueba de Kolmogorov-Smirnov para una muestra

\begin{tabular}{lrrrrrrr}
\hline & Promaprobadas & Puntaje & PromEGEL & PEHES & CEHS & AGC & PA \\
\hline $\mathbf{N}$ & 109 & 109 & 109 & 109 & 109 & 109 & 109 \\
$\begin{array}{l}\text { Media } \\
\begin{array}{l}\text { Desviación } \\
\text { típica }\end{array}\end{array}$ & 81.9029 & 1165.92 & 945.9335 & 972.367 & 942.678 & 989.018 & 879.669 \\
$\begin{array}{l}\text { Z de } \\
\text { Kolmogorov- } \\
\text { Smirnov }\end{array}$ & 3.55264 & 88.996 & 56.45993 & 80.165 & 72.9902 & 77.95678 & 86.64210 \\
$\begin{array}{l}\text { Sig. asintót. } \\
\text { (bilateral) }\end{array}$ & 1.211 & .518 & .679 & 1.153 & .807 & .870 & .872 \\
\hline
\end{tabular}

Fuente: Elaboración propia mediante utilización de programa IBM SPSS

\section{Variables relacionadas al rendimiento en el examen general de egreso}


\|lustración 2. Ejemplos de distribución de las variables PromEGEL, Puntaje y Promedio
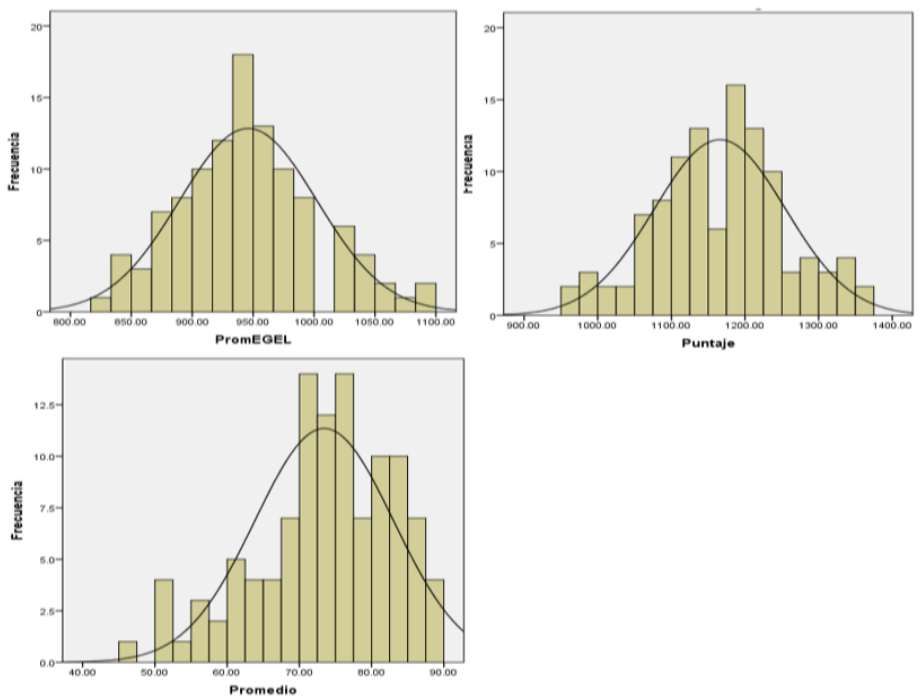

Fuente: Elaboración propia mediante utilización de programa IBM SPSS

Mediante la prueba de ANOVA (Tabla 2), se obtuvieron las significancias de cada variable en cuanto al test de Levene, el cual nos muestra valores $p>0.05$ suponiendo varianzas iguales, en caso que sean valores $p<$ 0.05 se suponen varianzas diferentes, esto nos ayuda a confirmar que la variabilidad de un grupo no sea distinta al del otro grupo. En caso de ser iguales se dice que se presenta homocestadicidad o homogeneidad de varianzas (Rubio Hurtado \& Berlanga Silvente, 2012, pág. 94). En este caso se compararon por el género y como podemos apreciar en la tabla solamente las variables Promaprobadas (.034) y CEHS (.043), mostrarón diferencias de variabilidad entre los grupos. Para la igualdad de medias se realizó la t de student resultando significativas las variables Promaprobadas (.005), CEHS (0.007) y ACG (.042).

Tabla 2 Prueba de ANOVA

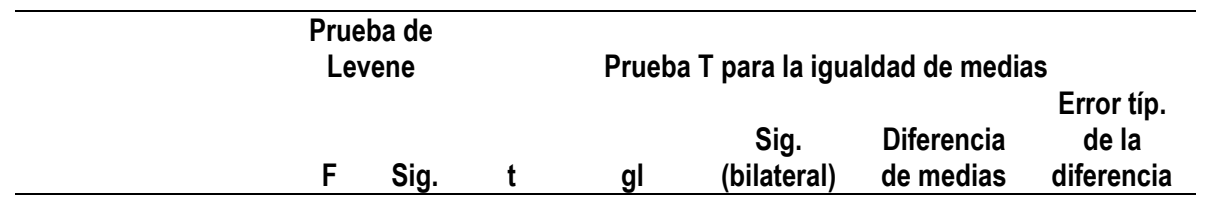

Fitch, M. \& Araiza, M. 


\begin{tabular}{lrrrrrrr} 
PromEGEL & .795 & .375 & 1.895 & 107 & .061 & 20.27969 & 10.69950 \\
& & & 1.880 & 99.940 & .063 & 20.27969 & 10.78638 \\
Promaprobadas & 4.630 & .034 & -2.877 & 107 & .005 & -1.89693 & .65943 \\
& & & -2.914 & 103.258 & .004 & -1.89693 & .65095 \\
Puntaje & .051 & .821 & -.697 & 107 & .487 & -11.92173 & 17.10736 \\
& & & -.696 & 105.184 & .488 & -11.92173 & 17.13812 \\
PEHES & 1.501 & .223 & .728 & 107 & .468 & 11.21323 & 15.40676 \\
& & & .726 & 104.699 & .469 & 11.21323 & 15.44657 \\
CEHS & 4.182 & .043 & 2.748 & 107 & .007 & 37.35189 & 13.59086 \\
& & & 2.717 & 96.235 & .008 & 37.35189 & 13.74575 \\
AGC & .073 & .788 & 2.057 & 107 & .042 & 30.30398 & 14.73075 \\
& & & 2.059 & 106.375 & .042 & 30.30398 & 14.71981 \\
\hline \multirow{2}{*}{ PA } & 1.058 & .306 & .135 & 107 & .893 & 2.24966 & 16.69115 \\
& & & .134 & 102.239 & .894 & 2.24966 & 16.78720 \\
\hline
\end{tabular}

Fuente: Elaboración propia mediante utilización de programa IBM SPSS

Estadistica descriptiva de los sustentantes

La muestra se encontró conformada por 109 estudiantes, 52 del género masculino y 57 femenino que presentaron examen de egreso en el semestre enero a junio del 2017. En cuanto a las variables del puntaje de examen de ingreso y los promedios de las unidades de aprendizaje cursadas, las mujeres obtuvieron ligeramente mejores resultados, sin embargo, en todas las áreas de evaluación del EGEL, los hombres superaron ligeramente a las mujeres. En cuanto a la experiencia profesional los hombres cuentan con más experiencia profesional que las mujeres.

Tabla 3. Variables y sus medias por género

\begin{tabular}{|c|c|c|c|c|c|c|}
\hline & \multicolumn{2}{|c|}{ Masculino (52) } & \multicolumn{2}{|c|}{ Femenino (57) } & \multicolumn{2}{|c|}{ Totales (109) } \\
\hline Variables & Media & $\begin{array}{c}\text { Desv. } \\
\text { típ. }\end{array}$ & Media & $\begin{array}{c}\text { Desv. } \\
\text { típ. }\end{array}$ & Media & $\begin{array}{c}\text { Desv. } \\
\text { típ. }\end{array}$ \\
\hline Puntaje & 1159.69 & 91.01 & 1171.61 & 87.54 & 1165.93 & 89.00 \\
\hline Promedio & 71.41 & 9.89 & 75.29 & 8.97 & 73.44 & 9.58 \\
\hline Promaterias & 79.23 & 4.05 & 80.38 & 4.92 & 79.83 & 4.55 \\
\hline
\end{tabular}

Variables relacionadas al rendimiento en el examen general de egreso 
180

\begin{tabular}{lrrrrrr} 
Promaprobadas & 80.91 & 2.90 & 82.81 & 3.86 & 81.90 & 3.55 \\
Expro & 3.56 & 1.09 & 3.05 & 1.11 & 3.29 & 1.12 \\
PEHES & 978.23 & 82.66 & 967.02 & 78.17 & 972.37 & 80.17 \\
CEHS & 962.21 & 79.53 & 924.86 & 61.95 & 942.68 & 72.99 \\
AGC & 1004.87 & 76.17 & 974.56 & 77.40 & 989.02 & 77.96 \\
PA & 880.85 & 92.54 & 878.60 & 81.70 & 879.67 & 86.64 \\
PromEGEL & 956.54 & 60.72 & 936.26 & 50.90 & 945.93 & 56.46 \\
\hline
\end{tabular}

Fuente: Elaboración propia mediante utilización de programa IBM SPSS

Con respecto a las 4 áreas se observó (Tabla 4) que la media de todas se posicionan en la clasificación de no satisfactorio. La evaluación del área de administración y gestión de la construcción (AGC) y el área de proyecto de espacios habitables con enfoque sustentable (PEHES), fueron las que obtuvieron medias y resultados más altos. Por el contrario, el examen práctico de proyecto arquitectónico $(\mathrm{PA})$ resulto con la media y las evaluaciones más bajas.

Tabla 4. Áreas de conocimiento EGEL

\begin{tabular}{lrrrrr}
\hline & \multicolumn{1}{c}{ Mínimo } & \multicolumn{1}{l}{ Máximo } & \multicolumn{1}{c}{ Media } & Desv. típ. & \multicolumn{1}{l}{ Varianza } \\
\hline PEHES & 828.00 & 1203.00 & 972.37 & 80.17 & 6426.57 \\
CEHS & 773.00 & 1166.00 & 942.68 & 72.99 & 5327.57 \\
AGC & 803.00 & 1185.00 & 989.02 & 77.96 & 6077.26 \\
PA & 719.00 & 1070.00 & 879.67 & 86.64 & 7506.85 \\
PromEGEL & 830.00 & 1099.25 & 945.93 & 56.46 & 3187.72 \\
\hline
\end{tabular}

Fuente: Elaboración propia mediante utilización de programa IBM SPSS

Para efectos de análisis de correlación entre variables numéricas se realizó el test de correlación de Pearson para todas las variables (tabla), para la interpretación de este coeficiente de contingencia de Pearson, Toscano y otros (2016), consideran que para un valor de 0 , se muestra nula relación entre variables; para valores mayores a 0 y menores a 0.2 , existe una mínima correlación; existe una correlación baja si el valor se encuentra mayor a 0.2 y menor a 0.4 ; si es mayor a 0.4 y menor a 0.6 , indica una correlación moderada; para valores mayores a 0.6 y hasta 0.8 , la correlación es buena; en caso de

\section{Fitch, M. \& Araiza, M.}


valores por encima de 0.8 , se considera muy buena; y cuando se presente un valor de 1, es una relación perfecta (pág. 75).

De acuerdo a los resultados (Tabla 5), las variables que guardan una correlación mínima positiva con los resultados del EGEL son el puntaje obtenido al ingreso, el grado académico de la madre y el promedio de la licenciatura (proaprobadas). Por otra parte, existe una correlación mínima negativa entre el promedio y la experiencia profesional.

Tabla 5. Correlaciones de Pearson para variables numéricas

\begin{tabular}{|c|c|c|c|c|c|c|c|c|c|c|c|c|}
\hline & $\begin{array}{l}\stackrel{\mathscr{W}}{\mathrm{I}} \\
\text { 岩 }\end{array}$ & $\begin{array}{l}\text { P } \\
\text { 岀 }\end{array}$ & 䜣 & 更 & 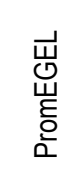 & 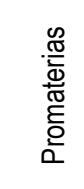 & 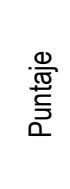 & $\begin{array}{l}\text { 응 } \\
\text { E } \\
\text { 음 } \\
0\end{array}$ & 을 & 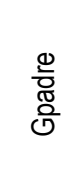 & $\begin{array}{l}\text { 這 } \\
\text { ¿ } \\
\text { E }\end{array}$ & 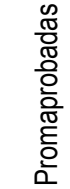 \\
\hline PromEGEL & 804 & .787 & .775 & .502 & 1 & .168 & .390 & .033 & .048 & .061 & 207 & $.232^{\circ}$ \\
\hline PEHES & 1 & .619 & .582 & .125 & .804 & $.222^{*}$ & .484 & .106 & -.021 & .006 & .234 & $.303^{\circ}$ \\
\hline CEHS & .619 & 1 & .573 & .121 & .787 & .083 & .247 & -.099 & .098 & .034 & . 140 & .128 \\
\hline AGC & .582 & .573 & 1 & .099 & .775 & .085 & .345 & .003 & .119 & .080 & 191 & .119 \\
\hline PA & .125 & .121 & .099 & 1 & .502 & .085 & .049 & .069 & -.046 & .065 & .036 & .109 \\
\hline Promate-rias & .222 & .083 & .085 & .085 & 168 & 1 & .161 & $.624^{* *}$ & $.263^{-}$ & .007 & $.049^{-}$ & .777 \\
\hline Puntaje & 484 & .247 & .345 & .049 & .390 & .161 & 1 & $.394^{* *}$ & -.100 & .076 & 119 & $.322^{\circ}$ \\
\hline Promedio & .106 & .099 & .003 & .069 & .033 & $.624^{4 *}$ & .394 & 1 & $.266^{-}$ & $.114^{-}$ & $.120^{-}$ & $.831^{\circ}$ \\
\hline Expro & .021 & .098 & .119 & .046 & .048 & $.263^{--}$ & $.100^{-}$ & $.266^{* *}$ & 1 & .034 & .106 & $.235^{\circ}$ \\
\hline Gpadre & .006 & .034 & .080 & .065 & .061 & -.007 & .076 & -.114 & -.034 & 1 & .462 & .006 \\
\hline Gmadre & .234 & .140 & .191 & .036 & .207 & -.049 & .119 & -.120 & .106 & .462 & 1 & .057 \\
\hline $\begin{array}{l}\text { Promaprobad } \\
\text { as }\end{array}$ & .303 & .128 & .119 & .109 & .232 & $.777^{7 *}$ & .322 & $.831^{* *}$ & $.235^{*}$ & .006 & $\begin{array}{r}- \\
.057\end{array}$ & 1 \\
\hline
\end{tabular}

${ }^{* \star}$. La correlación es significativa al nivel 0,01 (bilateral).

*. La correlación es significante al nivel 0,05 (bilateral).

Fuente: Elaboración propia mediante utilización de programa IBM SPSS

\section{Variables relacionadas al rendimiento en el examen general de egreso}


Por otro lado, la variable Promaterias, la cual está relacionada con aquellas UA que su contenido curricular está relacionado con la preparación del EGEL, solamente tiene correlación con el área PEHES (0.222). Además, de las cuatro áreas de conocimiento del EGEL, solamente PA no mantiene una correlación con el puntaje de acceso y a su vez esta área de conocimiento no conlleva una correlación con las otras tres áreas.

En el caso de las variables que no mostraron una distribución normal, homogeneidad de varianzas o que no eran del tipo numéricas se realizó la versión no paramétrica de rho de Spearman (Tabla 6), resultando correlacionadas el promedio del EGEL y el promedio de la licenciatura $(r=.280$, $p=.003)$, además, existe una correlación significativa entre el promedio de la licenciatura y la experiencia profesional $(r=-243, p=011)$.

Tabla 6. Rho de Spearman para datos no paramétricos

\begin{tabular}{|c|c|c|c|c|c|c|c|c|}
\hline & & $\begin{array}{c}\text { PromEGE } \\
L\end{array}$ & $\begin{array}{c}\text { Promaprobad } \\
\text { as }\end{array}$ & $\begin{array}{c}\text { CEH } \\
S\end{array}$ & AGC & $\begin{array}{c}\text { Expr } \\
0\end{array}$ & $\begin{array}{c}\text { Gpadr } \\
\text { e }\end{array}$ & $\begin{array}{c}\text { Gmadr } \\
e\end{array}$ \\
\hline \multirow[t]{2}{*}{ PromEGEL } & $\begin{array}{l}\text { Coeficient } \\
\text { e de } \\
\text { correlació } \\
n\end{array}$ & 1.000 & $.280^{* *}$ & $.724^{* *}$ & $.787^{*}$ & .017 & -.090 & .170 \\
\hline & $\begin{array}{l}\text { Sig. } \\
\text { (bilateral) }\end{array}$ & & .003 & .000 & .000 & .864 & .353 & .079 \\
\hline \multirow[t]{2}{*}{$\begin{array}{l}\text { Promaprobad } \\
\text { as }\end{array}$} & $\begin{array}{l}\text { Coeficient } \\
\text { e de } \\
\text { correlació } \\
n\end{array}$ & $.280^{* *}$ & 1.000 & .113 & .130 & $-.243^{*}$ & -.023 & -.074 \\
\hline & $\begin{array}{l}\text { Sig. } \\
\text { (bilateral) }\end{array}$ & .003 & & .241 & .178 & .011 & .814 & .444 \\
\hline \multirow[t]{2}{*}{ CEHS } & $\begin{array}{l}\text { Coeficient } \\
\text { e de } \\
\text { correlació } \\
n\end{array}$ & $.724^{* *}$ & .113 & 1.000 & $.566^{*}$ & .070 & -.110 & .110 \\
\hline & $\begin{array}{l}\text { Sig. } \\
\text { (bilateral) }\end{array}$ & .000 & .241 & & .000 & .468 & .256 & .259 \\
\hline \multirow[t]{2}{*}{ AGC } & $\begin{array}{l}\text { Coeficient } \\
\text { e de } \\
\text { correlació } \\
n\end{array}$ & $.787^{\star *}$ & .130 & $.566^{* *}$ & $\begin{array}{c}1.00 \\
0\end{array}$ & .107 & -.106 & .131 \\
\hline & $\begin{array}{l}\text { Sig. } \\
\text { (bilateral) }\end{array}$ & .000 & .178 & .000 & & .268 & .274 & .176 \\
\hline
\end{tabular}

Fitch, M. \& Araiza, M. 


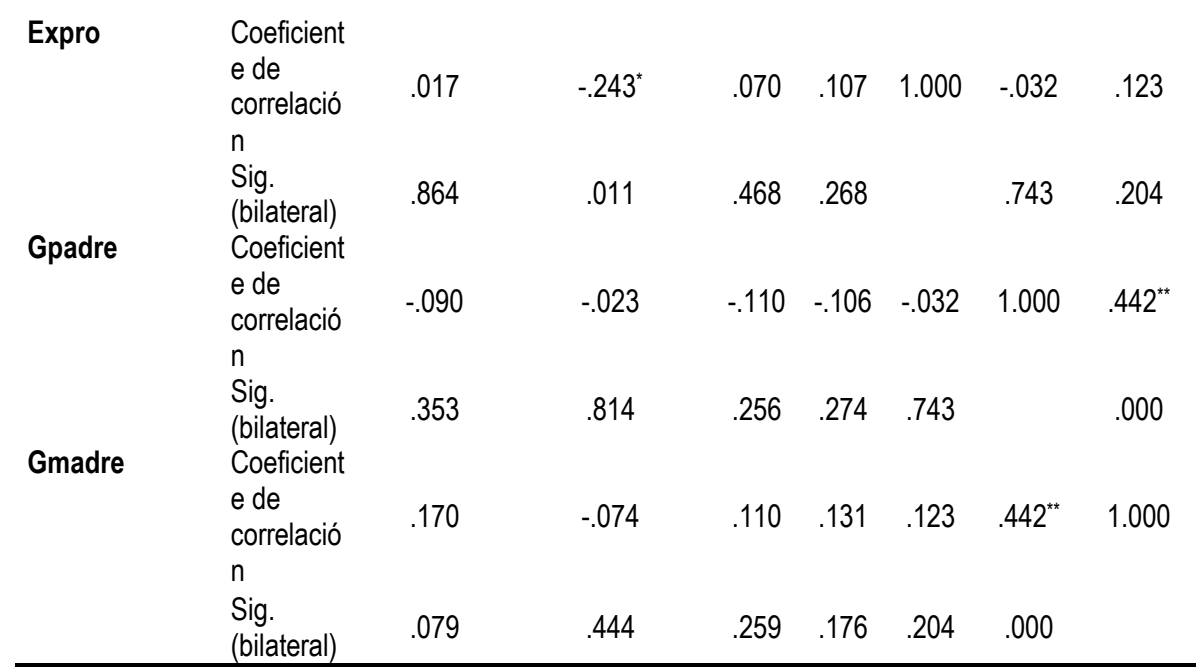

La correlación es significativa al nivel 0,01 (bilateral).**

La correlación es significativa al nivel 0,05 (bilateral).*

Fuente: Elaboración propia mediante utilización de programa IBM SPSS

\section{Discusión}

En cuanto a los resultados de las 4 dimensiones del examen observamos que el examen práctico PA, no guarda una correlación con las otras dimensiones, no se encuentra correlacionada con las UA que sirven como preparación para el EGEL, además que en esta evaluación se obtienen los resultados más bajos; De hecho, solamente la materia de taller integral II, está relacionada con las áreas de conocimiento PEHES y CEHS. En este orden de ideas, podemos suponer que algo le está afectando al estudiante en este tipo de evaluaciones y no es solamente la ausencia de conocimientos, esto deja un área de oportunidad para la academia, ya que debe de proponer más exámenes del tipo práctico similares a la prueba del EGEL, a fin que el alumno este más familiarizado con la dinámica y obtenga mayor experiencia en este tipo de pruebas.

Con respecto a la diferencia de resultados por género, las mujeres iniciaron su trayectoria académica a nivel superior, con un mejor puntaje en el examen de selección y alcanzaron mejor promedio durante su trayectoria

\section{Variables relacionadas al rendimiento en el examen general de egreso}


académica, sin embargo los hombres resultaron mejor evaluados en las pruebas de EGEL, resultando algo similar en lo presentado por Elías y otros (2016), señalando que los hombres son mejor evaluados en pruebas estandarizadas de manera objetiva, a diferencia de evaluaciones que han sido construidas en parte por valoraciones emitidas por el profesor (pág. 85), concordando también con Espinoza, Gómez y Suárez (2017), donde se evaluaron 32 programas educativos y los hombres obtuvieron mayor éxito. A manera de explicación podemos entender que el promedio se construye no solamente con las evaluaciones en exámenes, sino además con la entrega de distintos trabajos, participación en clase y demás actividades donde las mujeres pudieran presentar un mayor compromiso que los hombres, es por ello que las mujeres cuentan con un promedio más alto, sin embargo a la hora de demostrar sus competencias en pruebas estandarizadas los hombres perciben una mejor recompensa o premio y/o los motiva más el demostrar sus habilidades cognitivas a diferencia de las mujeres.

Referente a las correlaciones existentes entre variables, el puntaje de acceso guarda una baja correlación con el promedio de las evaluaciones del EGEL (.390), muy particularmente con las evaluaciones teóricas (PEHES .484, CEHS .247, AGS .345), además con el promedio obtenido, a lo que se puede considerar como una variable de tendencia de buen desempeño, resaltando que hay una predisposición a que los buenos estudiantes desarrollen un buen promedio y obtengan buenos resultados en los exámenes teóricos de EGEL, sin embargo requieren obtener otro tipo de competencias para lograr mejores resultados en el examen práctico.

La variable de grado de estudio de la madre, existe una baja correlación con el promedio del EGEL (.207), es congruente con lo señalado por Chaparro, González y Caso (2016), esta variable contribuye a la formación del perfil del estudiante, ya que al contar las madres con un mayor nivel educativo, estas cuentan con mejores hábitos de estudio para apoyar a sus hijos en los niveles básicos educativos y que los estudiantes conservan en los posteriores niveles, además propiciaron un clima cultural intelectual, el cual favoreció el desarrollo de mejores habilidades académicas y cognoscitivas (pág. 63). 
Por su parte la relación existente entre la experiencia profesional y las distintas variables del promedio, (promaterias -.263), (promedio -266), (promaprobadas -.235) las cuales presentaron bajas correlaciones negativas, nos indica que la exigencia de la carrera requiere cierta dedicación y que el trabajar y estudiar afecta al rendimiento académico del estudiante, por su parte, no hay evidencia que la experiencia profesional ayude a los resultados de las evaluaciones del EGEL.

\section{Conclusiones}

Referente a las hipotesis plateadas y las correlaciones que guardan entre variables hacia los resultados del examen de perfil de egreso, podemos decir lo siguiente:

El resultado del examen de ingreso a la UANL tiene una correlación significativamente positiva con el promedio de las cuatro áreas del EGEL, a manera individual, y teniendo como excepción del área de proyecto arquitectónico (PA), todas las áreas cuentan con una correlación positiva.

Se confirma que el promedio general en la licenciatura (Promaprobadas) tiene una correlación significativamente positiva con el promedio de las cuatro áreas del EGEL, sin embargo, a manera individual solamente con el área de PEHES resulto correlacionada.

El Promedio materias relacionadas con EGEL (Promaterias) no tiene una correlación el promedio de los resultados del perfil de egreso, solamente con el área de PEHES resulto correlacionada.

El Promedio (Promedio), no tiene una correlación con ninguno de los resultados del perfil de egreso.

El grado de estudios del padre no tiene una correlación no tiene una correlación con ninguno de los resultados del perfil de egreso.

El grado de estudios de la madre no tiene una correlación con el promedio de los resultados del perfil de egreso, solamente con el área de PEHES y AGC resulto correlacionada.

El Tiempo experiencia profesional relacionado con la licenciatura (Expro) no tiene una correlación con ninguno de los resultados del perfil de egreso.

\section{Variables relacionadas al rendimiento en el examen general de egreso}


De esta manera podemos observar que el área de conocimiento de proyecto Arquitectónico es la que requiere un mayor enfoque y suponemos que algunas de las causas se deben a que los temas de los exámenes prácticos en ocasiones se aplican en contextos de diferentes zonas del país, donde las condiciones geográficas, climáticas y demás factores, no son los que normalmente atiende el alumno en sus proyectos dentro de su trayectoria académica, por otro lado, los temas que se pueden llegar a abordar en el EGEL son muy diversos a lo que representa una complicación para los estudiantes, ya que existe la posibilidad que no lo presentaron previamente y no se encuentran familiarizados, lo que implica que una mayor complejidad al momento de presentar la evaluación.

Por último, el nivel de investigación no es suficiente para alcanzar explicaciones que determinen el grado de que influyen las variables en los resultados del mismo, a lo que se deberán abordar en futuras investigaciones con otras variables relacionadas al tema 0 ampliar la investigación a otros contextos donde se pretenda abordar el fenómeno a nivel predictivo.

Para futuras investigaciones es recomendable tomar en cuenta otras variables que nos puedan ofrecer información relacionada con los contenidos del EGEL, contrastándolo con el contenido de la curricula de la licenciatura de Arquitectura. Además, podría ser de utilidad considerar los resultados de las pruebas psicopedagógicas a las que se someten los estudiantes al inicio de la carrera en la Facultad de Arquitectura, como se trata del examen de inteligencia BAT-7 que evalúa las aptitudes cognitivas y el cual incorpora tres índices, capacidad general, inteligencia fluida e inteligencia cristalizada. Todo esto con la finalidad de encontrar correlaciones entre la inteligencia, el rendimiento escolar, así como los exámenes de egreso.

Resultaría interesante correlacionar los resultados del examen de personalidad TPT, el cual consta de 15 escalas en cuatro grandes dimensiones (estabilidad emocional, apertura mental, sociabilidad y responsabilidad) un índice de éxito en la vida profesional y una escala de sinceridad.

Fitch, M. \& Araiza, M. 
Sugerencias

En este orden de ideas y analizando los resultados con académicos de la Facultad de Arquitectura se pretenden desarrollar estrategias enfocadas con generar experiencia en evaluaciones prácticas las cuales se explican a continuación.

- Aumentar la frecuencia de ocasiones que el alumno desarrolle proyectos similares al EGEL, en condiciones y manera de evaluar similares, una ocasión por semestre, iniciando posiblemente desde séptimo u octavo semestre, cambiando la temática de los proyectos.

- Mediante pláticas de alumnos que anteriormente obtuvieron notables resultados en el EGEL, compartan sus experiencias y recomendaciones.

- Realizar pláticas con expertos en temas orientados a ampliar la visión del estudiante en cuanto a temas que apliquen en diferentes contextos y no solo que se quede con las situaciones que se resuelven a nivel regional.

- Incluir en el plan de estudios una unidad de aprendizaje enfocada a la dinámica del EGEL, que sirva de manera de guía o apoyo.

- Entregar a los estudiantes una rúbrica para la entrega de sus proyectos de octavo, noveno y décimo semestre, la cual es muy similar al nivel de exigencia del examen del EGEL.

- Revisar la malla curricular para revisar las UA y su impacto al EGEL, procurando que el estudiante reciba temas básicos de fondo donde se le otorguen las herramientas para analizar a profundidad el contexto y la problemática a resolver.

\section{Referencias}

Bandura, A. (1977). Self-efficacy: Toward a Unifying Theory of Behavioral Change. Psychological Review, 84(2), 191-215.

Castillo Ramírez, A., Izar Landeta, J. M., \& Espericueta González, D. E. (2013). Correlación entre trayectoria académica y el examen nacional de egreso de la licenciatura. Revista de Investigación Educativa, 172-188.

\section{Variables relacionadas al rendimiento en el examen general de egreso}


Centro Nacional de Evaluación para la Educación Superior, A. (2018). Informe Anual de Resultados 2017 (EGEL-ARQUI). Retrieved Noviembre 17, 2018, from http://www.ceneval.edu.mx/estadisticas-ceneval

Centro Nacional para la Educación Superior, A.C. (2019). Guía EXANI II. 24a. México: CENEVAL.

Chaparro Caso López, A. A., González Barbera, C., \& Caso Niebla, J. (2016). Familia y rendimiento académico: configuración de perfiles estudiantiles en secundaria. Revista Electrónica de Investigación Educativa, 18(1), 53-68.

Cortés Flores, A., \& Palomar Lever, J. (2008). El proceso de admisión como predictor del rendimiento académico en la educación superior. Universitas Psychologica, 197-213.

Elías Jimenez, C. I., Calderas Montes, J. F., Reynoso González, O. U., \& Zamora Betancourt, M. d. (2016). Variables asociadas al rendimiento en el Examen General para el Egreso de Licenciatura. El caso de Psicología. Revista de la Educación Superior, 75-88.

Espinoza Ortega, O., Gómez Vidal, A., \& Suarez Varela, I. (2017). Análisis del resultado del Examen General de Egreso de Licenciatura (EGEL) bajo un enfoque de género, considerando los sustentantes y los que diseñan dicho instrumento de evaluación. Debates en Evaluación y Currículum/Congreso Internacional de Educación, 1-10. Retrieved from http://posgradoeducacionuatx.org/pdf2017/D007.pdf

Guerra Moya, S. A., \& Ponce Sánchez, R. (2014). Análisis Multivariante: Modelización con ecuaciones estructurales. In K. Sáenz López, \& G. Tamez González, Métodos y Técnicas Cualitativas y Cuantitativas Aplicables a la Investigación en Ciencias Sociales (pp. 316-350). México D.F.: Tirant Humanidades México.

Guerrero Ávila, J. J., Romero Gónzalez, J., \& Noriega, R. (2013). Análisis de la competencia en medicina visto a través del EGEL-MG. CULCyT, 2636.

Izar Landeta, J. M., \& López Gama, H. (2009). El puntaje que obtienen los estudiantes de Contaduría Pública en el examen general de egreso de 
la licenciatura (EGEL) y su relación con el promedio obtenido durante su carrera. Hitos de ciencias económico administrativas, 27-34.

Ornelas, M., Blanco, H., Rodríguez, J., \& Flores, F. (2011). Análisis Psicométrico de la Escala Autoeficacia en Conductas de Cuidado de la Salud Física en Universitarios de Primer Ingreso. Formación Universitaria, 4(6), 21-34. doi:10.4067/S0718-50062011000600004

Rubio Hurtado, M. J., \& Berlanga Silvente, V. (2012). Cómo aplicar las pruebas paramétricas bivariadas $t$ de Student y ANOVA en SPSS. Caso práctico. REIRE, Revista d'Innovació i Recerca en Educació, 5(2), 83100. Retrieved from http://www.ub.edu/ice/reire.htm

Toscano de la Torre, B. A., Ponce Gallegos, J. C., Margain Fuentes, M., \& Vizcaíno Monroy, O. G. (2016). Estudio exploratorio de los resultados del EGEL-I-CENEVAL como base para identificar factores que determinan su acreditación. Educateconciencia, 64-82.

Variables relacionadas al rendimiento en el examen general de egreso 